\title{
Clot injection for treatment of iatrogenic femoral arteriovenous fistula after percutaneous coronary intervention: a novel minimally invasive method
}

\author{
Jacek Kurzawski ${ }^{1}$, Marcin Sadowski ${ }^{1,2}$, Marianna Janion $^{1,2}$ \\ ${ }^{1} 2^{\text {nd }}$ Department of Cardiology, Świętokrzyskie Cardiology Centre, Kielce, Poland \\ ${ }^{2}$ Institute of Medical Sciences, Medical Faculty and Health Sciences, Jan Kochanowski University, Kielce, Poland
}

Adv Interv Cardiol 2016; 12, 4 (46): 364-367

DOI: 10.5114 /aic.2016.63638

\section{Introduction}

latrogenic arteriovenous fistula (AVF) is usually caused by inappropriate blood vessel cannulation during diagnostic and interventional procedures [1-3]. Arteriovenous fistulas occur in $1.0-1.5 \%$ of cases following procedures with femoral access (FA) $[1,4,5]$. latrogenic fistulas are unintentional connections between an artery and a vein due to the needle being advanced too deeply and reaching the vein lumen, resulting in a constant flow of blood between the artery and the vein. Arteriovenous fistulas sometimes coexist with pseudoaneurysms (PAS) $[6,7]$. Arteriovenous fistulas are characterized by a high-gradient blood flow through the fistula from artery to vein (Figures $1 \mathrm{~B}$ and C). There are three types of AVF. The most frequent type is an AVF in which the fistula is only an anastomosis between the lumen of the artery and the vein. Another type is a pseudochannel varying in length between the two vessels (Figures $1 \mathrm{~A}$ and $\mathrm{C}$ ). The third type is an AVF (a channel or anastomosis) with an accompanying PAS. Several therapeutic strategies have been developed to treat iatrogenic arteriovenous fistulas. Most frequently, but not in all cases, it is insertion of a stent graft $[8,9]$. A rare occurrence of this complication may be the main obstacle in developing simple treatment strategies, and even large-volume interventional centres use different approaches to treat AVFs. Bearing in mind the advantages and disadvantages of the available strategies, we developed a simple, cost-effective and safe technique to treat an arteriovenous fistula with a channel-type connection.

\section{Material and methods \\ This experimental study was approved by the Bioeth- ics Committee of the Local Chamber of Physicians. Writ-}

ten informed consent was obtained from each patient after a detailed explanation of the procedure. All patients with a channel-type AVF were followed up for 3 weeks after the initial diagnosis. Those who had the AVF patent underwent AVF closure. A total of 6 patients were included. Basic clinical characteristics are summarized in Table I. In 4 cases AVF was between the superficial femoral artery and the great saphenous vein, and in 2 cases it was between the common femoral artery and the great saphenous vein. The length of the connection ranged from 30 to $55 \mathrm{~mm}$, and it had a tortuous course. During Doppler ultrasound the proximal end of the connection, i.e. the outflow of blood from the artery to the fistula (Figure $1 \mathrm{~B}$ ), and the distal end, i.e. the outflow of blood from the fistula to the vein (Figure $1 \mathrm{C}$ ), were indentified. The idea behind our technique is to perform a compression near the fistula using biological materials with density higher than the density of physiological saline. First, the patient's blood was collected into a $5 \mathrm{ml}$ syringe. A dose of $20 \mathrm{IU}$ of bovine thrombin solution was drawn into another $5 \mathrm{ml}$ syringe, which was then filled with the patient's blood from the first syringe to initiate the formation of a clot, which was ready to use in a short time. Needles had a minimum cross diameter of $1 \mathrm{~mm}$; otherwise application of the clot might have been difficult. Blood clots were prepared immediately before injection when they were very elastic and easy to apply with a needle. The clots were injected at the site most distant from the neighbouring blood vessels and closest to the fistula (Figure $1 \mathrm{E}$ ) within several minutes after mixing thrombin with blood. The location of the needle tip was monitored during the procedure by moving the needle with short vibrating movements. The volume of the injected clot was equivalent to about $5 \mathrm{ml}$ of blood prior to coagulation. 

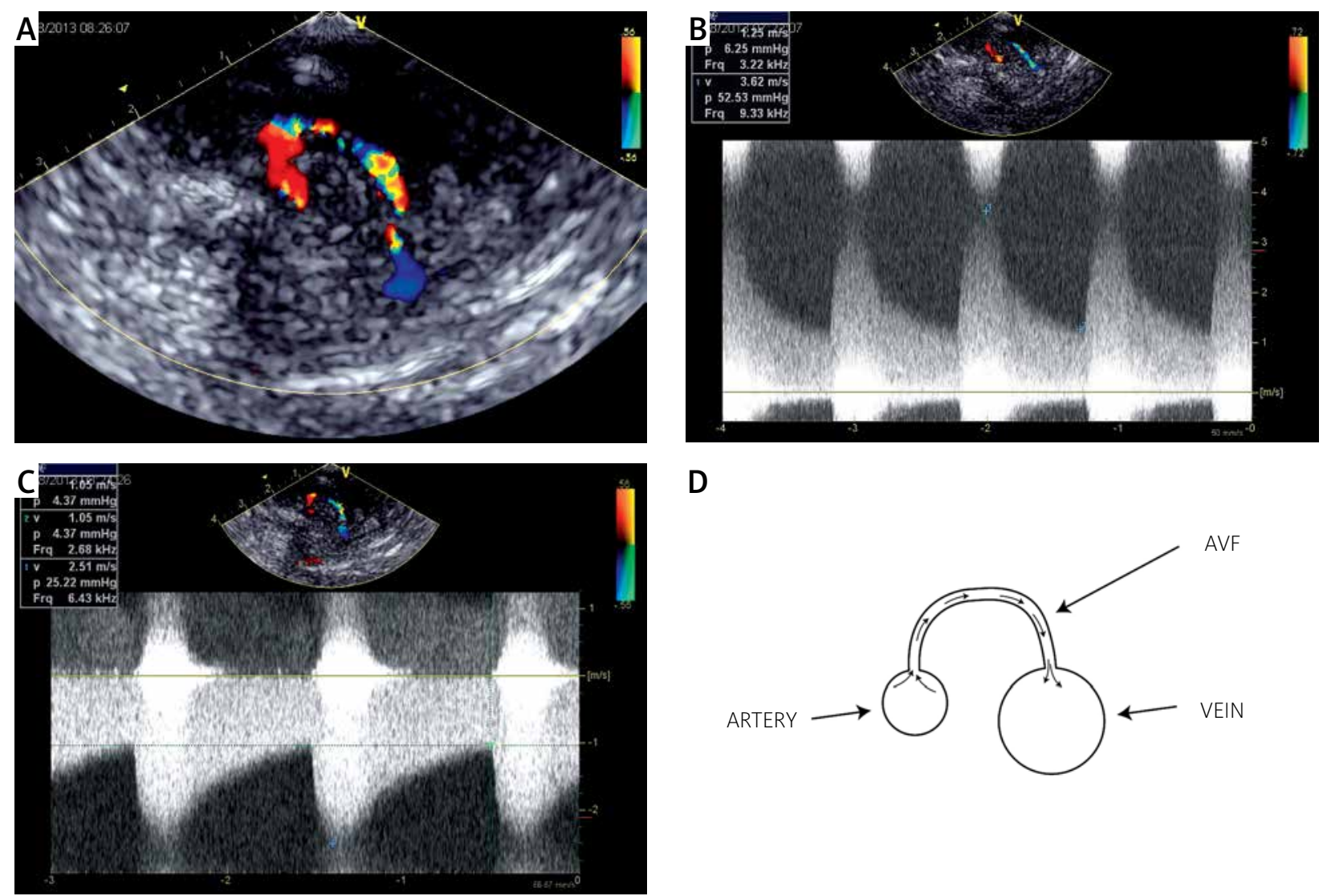

D

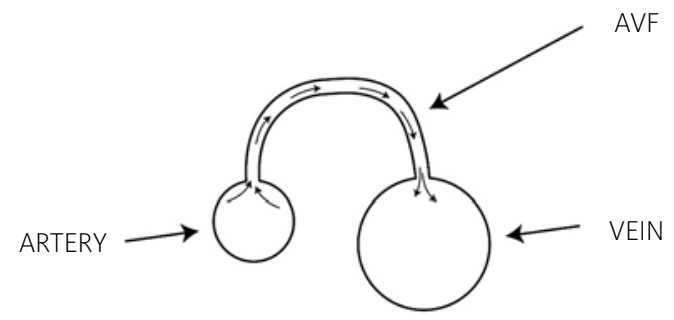

E

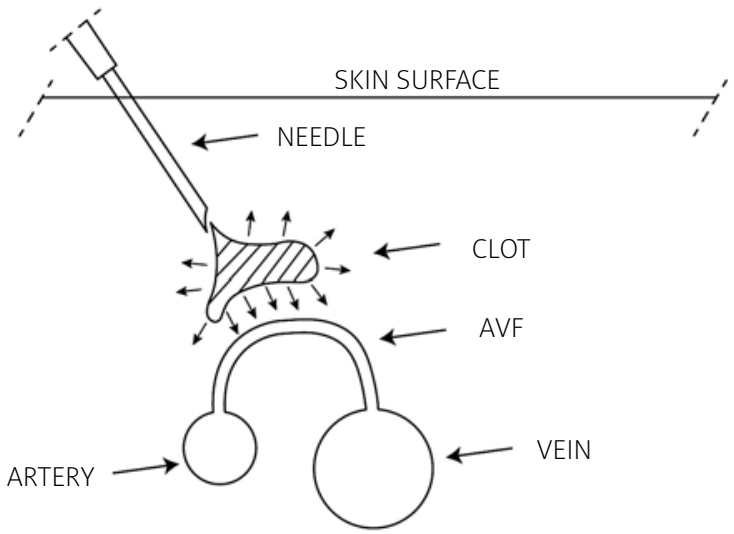

$\mathrm{F}$

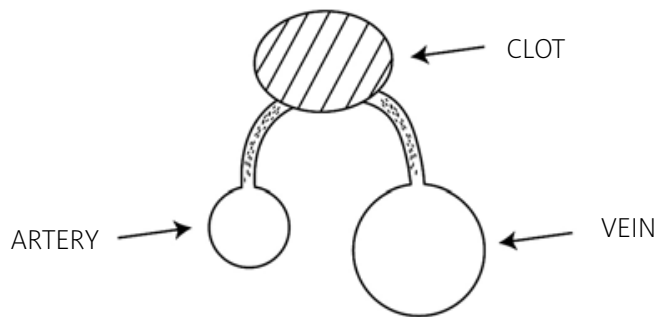

Figure 1. A-C - Colour Doppler ultrasound examination. Arteriovenous fistula with channel (A). Continuous wave Doppler arterial (B) and venous (C) end flow. D - Arteriovenous fistula as in Panel A. Clot injection (E) and fistula closure (F)

Table I. Baseline clinical characteristics

\begin{tabular}{|c|c|c|c|c|c|}
\hline Gender & Age [years] & Channel length [mm] & Arterial flow [m/s] & Fistula flow $[\mathrm{m} / \mathrm{s}]$ & Presentation \\
\hline$F$ & 57 & 55 & 0.9 & $3.15-1.3$ & Stable \\
\hline M & 70 & 31 & 0.9 & $3.16-2.1$ & Acute \\
\hline $\mathrm{F}$ & 68 & 30 & 0.9 & $5.3-2.8$ & Stable \\
\hline $\mathrm{F}$ & 61 & 33 & 0.8 & $2.7-1.9$ & Acute \\
\hline $\mathrm{F}$ & 67 & 35 & 1.02 & $2.94-1.02$ & Acute \\
\hline $\mathrm{F}$ & 80 & 42 & 0.92 & $4.3-1.58$ & Acute \\
\hline
\end{tabular}

F-female, $M$ - male, acute/stable - procedure for acute coronary syndrome/stable angina. 
Using $5 \mathrm{ml}$ syringes we performed one or two injections. Clot injection near the fistula caused compression on the neighbouring tissues, including the AVF channel, leading to obliteration of the lumen and cessation of blood flow (Figure $1 \mathrm{~F}$ ) depicted as a cross section of the artery and vein and no flow in colour Doppler imaging. Blood flow cessation resulted in spontaneous thrombosis in the remaining section of the fistula.

\section{Results}

All clot injections resulted in fistula closure without complications. Each patient reported a slight distension sensation at the injection site. No inflammatory reaction was observed. The 12-month follow-up was uneventful.

\section{Discussion}

Complications associated with femoral access, irrespective of their morphological type, always pose a problem, especially when occurring sporadically. Arteriovenous fistula is a rare complication, especially AVF with a pseudochannel. Unfortunately, most iatrogenic AVFs occur in patients after cardiac catheterization [10, 11]. Some patients, especially after interventional procedures, must receive antiplatelet agents. For this reason surgical treatment is associated with increased risk of bleeding. Endovascular stent graft placement is another recommended treatment strategy [9]. Despite expected benefits, this strategy is associated with a significant risk of thrombosis; therefore stent grafts are used only when the AVF communicates with a large artery such as the femoral artery. Other techniques include coil implantation and percutaneous injection of substances to obliterate fistulas [12]. Direct mechanical compression using the ultrasound probe or pressure dressing was attempted previously [8]; however, due to several drawbacks (long procedure time, discomfort, superficial thrombophlebitis risk) [13] it is no longer applied. Our strategy of ultrasound-guided clot injection near the fistula may be worth considering in patients with AVF and a distinct connection channel. It is simple, cheap, effective and patient friendly. This technique for AVF closure has not been described in the literature, although treatment with injections of physiological saline solution has already been reported. The procedure of internal compression was attempted in patients with pseudoaneurysms, but it has not gained popularity. In our centre we have made two attempts to treat AVF using internal compression with physiological saline. However, the procedure was unsuccessful and painful for the patients because of high volume saline injections. The reason was low density of saline solution, which easily diffused into surrounding subcutaneous tissue and did not produce effective compression. Moreover, the volume of saline solution is several times larger than the volume of the injected clot. Finally, Toursarkissian demonstrated that about $1 / 3$ of iatrogenic fistulas may close spontaneously within a year $[14,15]$. It is a very positive finding, but we never know whether the fistula will have the chance to heal spontaneously when the patient is discharged with the complication. "A small AVF" is a relative term, as its true lumen size is very difficult to assess, and its left-to-right shunt is usually insignificant. However, the negative impact on the venous system has not been investigated previously. Moreover, a complication left untreated may develop into a more serious condition. Therefore, all patent AVF were referred for the closing procedure.

A separate problem is the absence of standard ultrasound criteria for fistula closure. The nature of tissues involved significantly affects imaging. AVFs do not have a regular hypoechogenic morphology (like aneurysms) and are not visible in two-dimensional ultrasound imaging. The needle used is thin and cannot be captured in a still image. The clot has echogenicity similar to the surrounding tissue, which represents mainly haemorrhagic infiltration. The only proof of the patent AVF is a high threshold colour flow (i.e. $96 \mathrm{~cm} / \mathrm{s}$ ). In contrast, the picture of a closed AVF is composed of the cross section of the artery and vein and no flow in colour Doppler imaging.

\section{Conclusions}

Clot injection for the treatment of iatrogenic femoral arteriovenous fistula seems to be a safe, cheap and feasible method.

\section{Conflict of interest}

The authors declare no conflict of interest.

\section{References}

1. Pitta SR, Prasad A, Kumar G, et al. Location of femoral artery access with vascular complications. Catheter Cardiovasc Interv 2011; 78: 294-9.

2. Kleczynski P, Rakowski T, Dziewierz A, et al. Ultrasound-guided thrombin injection in the treatment of iatrogenic arterial pseudoaneurysms: single-center experience. J Clin Ultrasound 2014; 42: 24-6.

3. Stone PA, Campbell JE. Complications related to femoral artery access for transcatheter procedures. Vasc Endovascular Surg 2012; 46: 617-23.

4. Ohlow MA, Seckuns MA, von Korn $\mathrm{H}$, et al. Incidence and outcome of femoral vascular complications among 18.165 patients undergoing cardiac catheterization. Int J Cardiol 2009; 135: 66-71.

5. Crudu V, Blankenship J, Berger P, et al. Complications related to access site after percutaneous coronary interventions: are the adverse events underreported? Catheter Cardiovasc Interv 2011; 77: 643-7.

6. Lønnebakken MT, Gerdts E, Pedersen OM. Femoral pseudoaneurysm with a communicating arteriovenous fistula: a complication after percutaneous coronary intervention. Circulation 2012; 126: e161-2.

7. Belli S, Parlakgumus A, Colakoglu T, et al. Surgical treatment modalities for complicated aneurysms and pseudoaneurysms of arteriovenous fistulas. J Vasc Access 2012; 13: 438-45. 
8. Tao Z, Zhen-Jiang L, Sheng-Hua Z, et al. Treatment of postcatheterization femoral arteriovenous fistulas with simple prolonged bandaging. Chin Med J 2007; 120: 952-5.

9. Bellmunt S, Dilme J, Barros A, et al. Compression assisted by removable coils as a new treatment for iatrogenic femoral pseudoaneurysms. J Vasc Surg 2011; 53: 236-8.

10. Tsetis D. Endovascular treatment of complications of femoral arterial access. Cardiovasc Intervent Radiol 2010; 33: 457-68.

11. Riley R, Don C, Powell W, et al. Trends in coronary revascularization in the United States From 2001 to 2009 recent declines in percutaneous coronary intervention volumes. Circ Cardiovasc Qual Outcomes 2011; 4: 193-7.

12. Onal B, Ilgit ET, Akpek S, et al. Postcatheterization femoral arteriovenous fistula: endovascular treatment with $\mathrm{N}$-butyl-cyanoacrylate embolization. Cardiovasc Intervent Radiol 2006; 29: 276-8.

13. De Santis F, Zywica M, Mani G, et al. Localized superficial femoral vein thrombosis coupled with iatrogenic arteriovenous fistulas presenting as pulmonary emboli. Phlebology 2012; 27: 191-3.

14. Kelm M, Perings $S$, Jax T. Incidence and clinical outcome of iatrogenic femoral arteriovenous fistulas: implications for risk stratification and treatment. J Am Coll Cardiol 2002; 40: 291-7.

15. Toursarkissian B, Allen B, Petrinec D, et al. Spontaneous closure of selected iatrogenic pseudoaneurysms and arteriovenous fistulae. J Vasc Surg 1997; 25: 803-8. 